\title{
Self-healing Graphene-based composites with sensing capabilities
}

Eleonora D’Elia, Suelen Barg ${ }^{1}$, Na Ni, Victoria G. Rocha \& Eduardo Saiz

Centre for Advanced Structural Ceramics, Department of Materials, Imperial College London, London SW7 2AZ, United Kingdom.

Natural systems are a rich source of scientific inspiration. Skin for example functions as an efficient protective barrier for the human body that is able to sense the external environment and repair autonomously. The translation of these physiological properties to synthetic materials could open new opportunities in many strategic fields from health care to robotics. In recent years, significant advances have been accomplished towards the development of synthetic materials with unique sensing and/or self-healing capabilities ${ }^{[1,2]}$. The ability to self-heal often relies on the use of an external stimulus to trigger repair ${ }^{[3]}$ or on the use of vascular [1,4] or capsule-based [5] systems for the storage and release of healants upon damage. However, these systems often show incomplete healing, cannot heal multiple times, or require the prompt location of the damage site. An alternative is the use of supramolecular polymers (macromolecular aggregates cross-linked by dynamic covalent or hydrogen bonds) that provide an efficient path towards autonomous multiple self-healing [6]. Still, the integration of healing ability with functional capabilities in robust and lightweight materials remains a challenge. In this work, we marry both approaches to develop robust, electrically conductive, self-healing composites. These composites, prepared through the encapsulation of a self-healing supramolecular polymer in a graphene ultralight network, are able to sense pressure and flexion and completely and 
autonomously restore their properties multiple times after damage and without the necessity of an external stimulus.

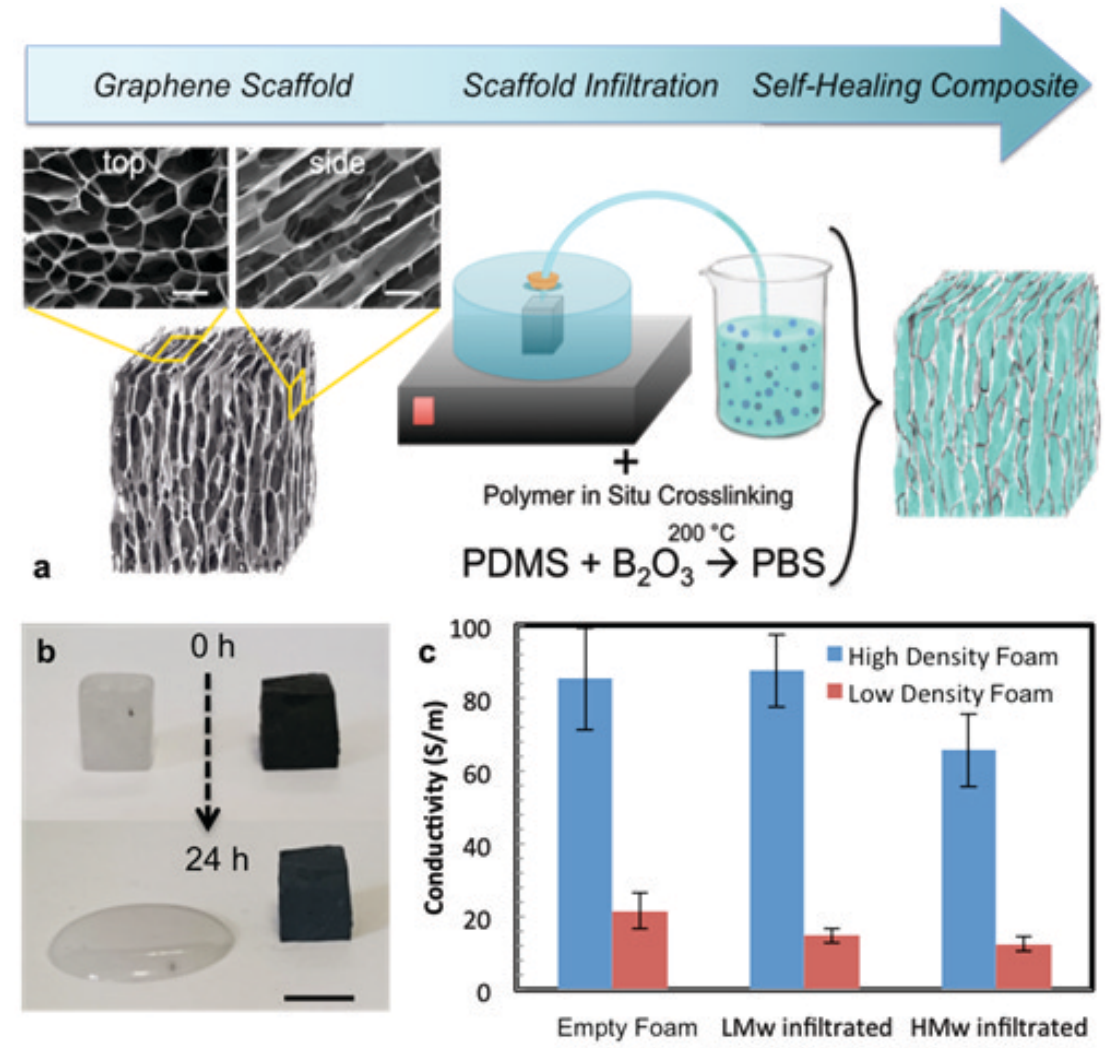

Figure 1: Processing, mechanical stability and electrical conductivity. (a) From left to right: Ultra-light reduced graphene oxide (rGO) networks formed by microscopic channels separated by thin walls and packed to form a honeycomb cross-section (scale bar $20 \mu \mathrm{m}$ ). The scaffolds are infiltrated with a $\mathrm{PDMS} / \mathrm{B}_{2} \mathrm{O}_{3}$ solution via vacuum casting. Cross-linking of the polymer into PBS takes place in-situ by heating the infiltrated networks at $200^{\circ} \mathrm{C}$. The resulting composites are composed of a rGO continuous network encapsulating PBS. Infiltration is complete and the residual porosity in the composite is less than $1 \%$. (b) Mechanical stability. Optical images showing the flow behavior of polymer-only (left) and composite samples (right) of equal size and shape. After only a few hours in air at room temperature the "solid-liquid" polymer flows due to its viscoelastic properties forming a film that spreads on the substrate whereas the composite formed by the polymer confined in the graphene network retains its structure (scale bar 1mm). (c) Effect of network density (low density, $2.5 \pm 0.2 \mathrm{mg}$ $\mathrm{cm}^{-3}$, high density $11 \pm 1 \mathrm{mg} \mathrm{cm}^{-3}$ ) and PBS molecular weight (Low: $25 \mathrm{cSt}$ (LMw) and High: $65 \mathrm{cSt}(\mathrm{HMw}))$ on the electrical conductivity of the composites. The conductivity can reach values up to $90 \mathrm{~S} \mathrm{~m}^{-1}$ for materials fabricated from highdensity networks as their graphene content is larger. Expansion of the network after polymer infiltration may reduce conductivity slightly. 
Polyborosiloxane (PBS) is a supramolecular polymer that exhibits an intrinsic selfhealing character via dynamic dative bonds (created via triple and quadruple bonds between Boron and the Oxygen in the Si-O groups). However, PBS is a well-known "solid-liquid" material whose viscoelastic properties (it flows as a highly viscous liquid at low strain rates but behaves as a solid at high strain rates) promote fast and complete healing but impair structural applications ${ }^{[7]}$. In order to impart electrical conductivity and increase strength we have used standard homogenization techniques to disperse reduced graphene oxide (rGO) flakes in PBS. The resulting material remains electrically insulating even with rGO contents as high as $10 \mathrm{wt} \%$. This agrees with previous calculations ${ }^{[8]}$ of the percolation threshold for high-aspect ratio platelets, and the problem increases by flake crumpling and agglomeration. In addition, the material still exhibits a shearthickening "solid-liquid" behavior with storage $(G$ ') and loss modulus (G") comparable to PBS $(\mathrm{SI})$.

To overcome these limitations we have encapsulated the self-healing polymer in microporous reduced graphene oxide ( $\mathrm{rGO}$ ) networks prepared through the freeze casting of graphene oxide (GO) slurries followed by thermal reduction [9]. The technique uses the directional freezing of the suspensions to form networks with a characteristic structure formed by long channels (hundred of microns) packed in a honeycomb arrangement. The channels, templated by the ice crystals, are aligned along the direction of ice growth. Their diameter is of the order of $\sim 10-20 \mu \mathrm{m}$ are they are separated by thin (below $50 \mathrm{~nm}$ ) carbon walls formed by an accumulation of entangled rGO flakes bonded together by physical forces (Fig. 1). The final densities of the networks range between 2 to $11 \mathrm{mg} \mathrm{cm}^{-}$ ${ }^{3}$, depending on the concentration of the starting GO suspensions. Their 
compressive yield strengths increase with density between 1 to $30 \mathrm{kPa}$. Due to their hydrophobic nature, the rGO scaffolds can be fully infiltrated with a solution of PDMS and boron oxide nanoparticles to form "in situ" the PBS polymer. The final rGO content of the composites is very low, and varies between 0.2 and 1wt.\%.
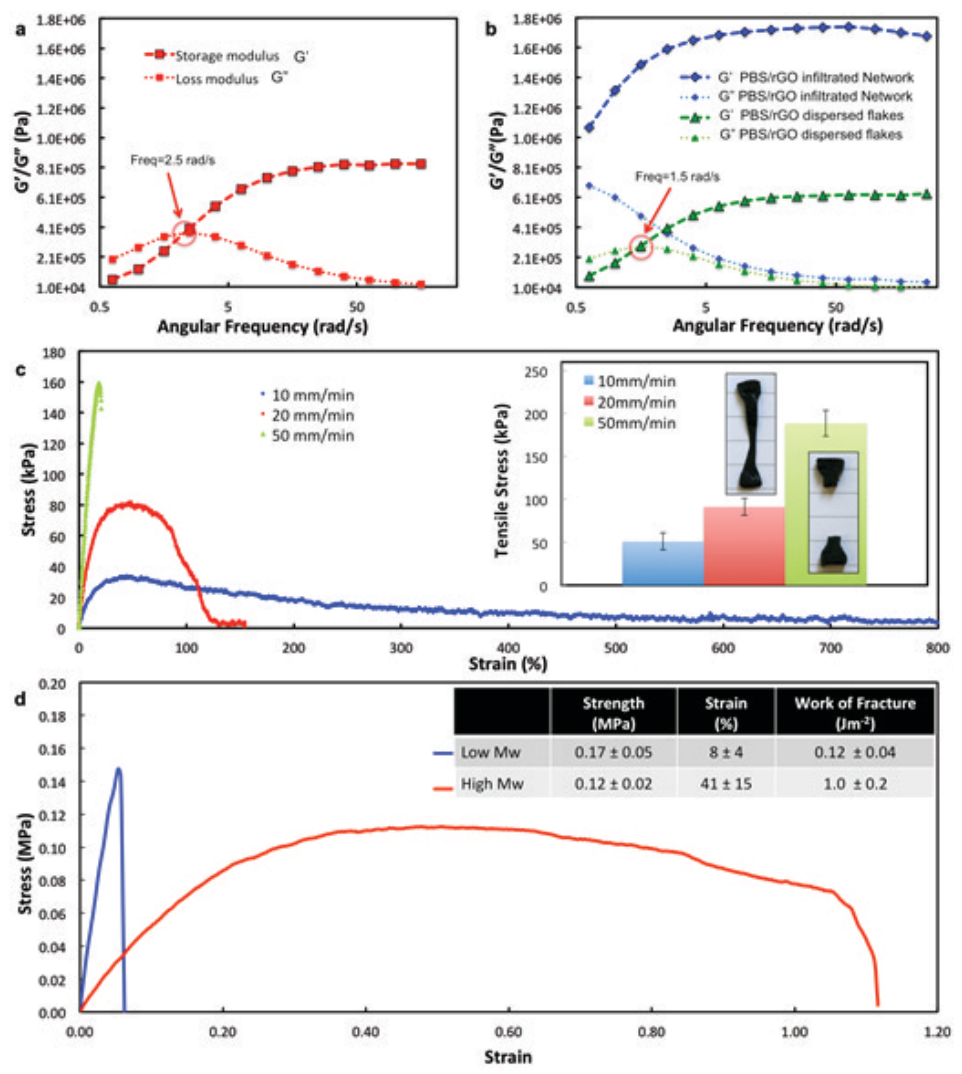

Figure 2: Mechanical performance. (a) Viscoelastic properties of PBS. The polymer exhibits a shear-thickening behavior, with the storage modulus ( $G$ ') surpassing the Loss modulus (G') at high frequencies (crossover point at 2.5 rads $^{-1}$ ). (b) Viscoelastic properties of the rGO scaffolds/LMw-polymer composite (in blue) and polymer with dispersed graphene flakes (in green) (rGO content 0.5 wt. $\%$ in both cases). While the composite produced by infiltrating a graphene network exhibits a solid-like behavior for all the frequency range, the composite produced by the dispersion of graphene in the polymer shows a shear-thickening behavior similar to the polymer itself (a), with a crossover point at $1.5 \mathrm{rads}^{-1}$. (c) Typical tensile stress/strain curves of the composites at different strain rates showing stronger responses and less elongation for higher rates. (c inset) Tensile strength as a function of strain rate. Dog-bone shape fractured samples (inset) show the change in the fracture behavior. (d) Typical stress/strain curves for composites made out with polymers of two different molecular weights (1000 vs $3500 \mathrm{Da}$ ). 
The rGO network acts as a skeleton that provides mechanical stability and encapsulates PBS impeding flow. The storage modulus of the composite is up to two orders of magnitude higher than PBS alone or with dispersed rGO flakes. More importantly, it has a solid behavior (G'>G') in the whole range of frequencies analyzed (Fig. 2 b). The graphene network also acts as a continuous electrical path providing electrical conductivities up to $90 \mathrm{~S} \mathrm{~m}^{-1}$ (Fig. 1c).

The tensile behavior of the composites is highly dependent on the strain rates and they can reach tensile strengths up to $0.2 \mathrm{MPa}$ at the faster rates $(50 \mathrm{~mm}$ $\min ^{-1}$, Figs. $2 c$ and $2 d$ ). The strain-dependence results from the shear-thickening behavior of the encapsulated PBS (Fig. 2). This is strongly dictated by the B-O dynamic covalent bonds that form the viscoelastic polymer network $[10,11]$. While at low strain rates the polymer flows as a viscous fluid (due to a more loose arrangement of the polymer segments) at fast strain rates the network gets tighter and the polymer becomes elastic, breaking in a more brittle manner [11,12]. When the test is carried out at lower rates (typically below $10 \mathrm{~mm} \mathrm{~min}^{-1}$ ) the composite exhibits large elongations to fracture due to the viscous behavior of the polymer. 

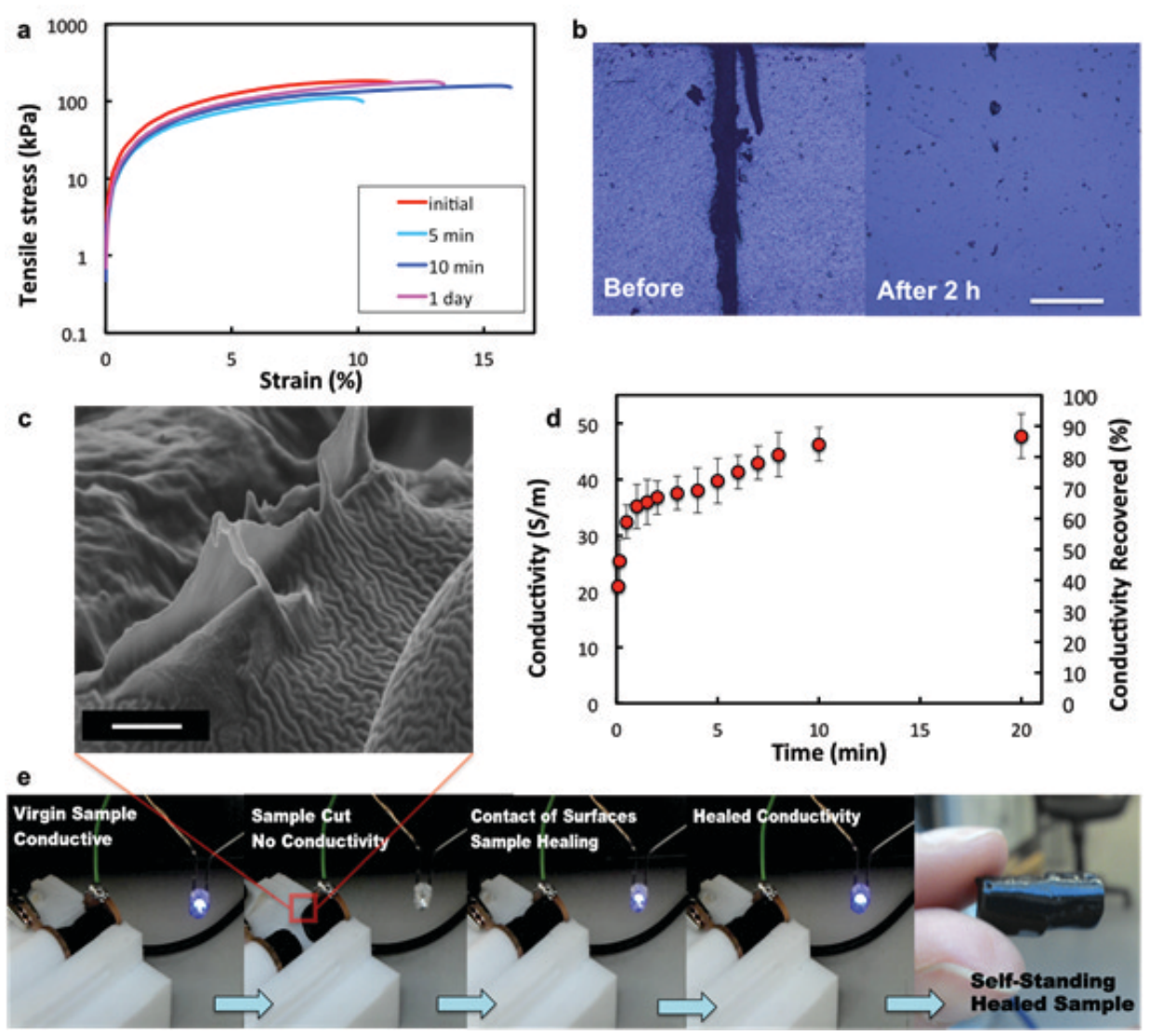

Figure 3: Electrical and mechanical healing. (a) Tensile stress-strain curves for original and healed samples after healing times ranging from $5 \mathrm{~min}$ up to 1 day. Tensile tests carried out at $50 \mathrm{~mm} \mathrm{~min}^{-1}$ strain rate on dog bone samples. (b) Optical microscopy image of a deeply scratched composite and its physical healing after 120 minutes (scale bar $1 \mathrm{~mm}$ ). (c) SEM image of a fractured interface showing how the PBS wets the rGO wall (scale bar $1 \mu \mathrm{m})$. (d) Recovery of electrical conductivity as a function of time for a sample with a starting conductivity of $55 \mathrm{~S} \mathrm{~m}^{-1}$ composed of 0.5 wt.\% graphene with LMw polymer. (e) Demonstration of electrical healing by placing the composite in series with a LED. From left to right: original composites showing the passage of current though the conductive sample (LED is on); the drop in current (open circuit) when the sample is cut in half (LED is off) and the gradual recovery after the two surfaces are put back into contact with no pressure (LED is on and recovers the original light intensity after 10 minutes).

The mechanical response depends on the molecular weight of the polymer (Fig.

$2 \mathrm{~d}$ inset). The use of a low molecular weight polymer (circa $1000 \mathrm{Da}$ ) results in a stiffer and more brittle material. On the other hand, even if showing lower maximum strength, a higher molecular weight polymer (circa $3500 \mathrm{Da}$ ) leads to 
values of work of fracture 10 times higher (Fig. 2). This is due to the intrinsic stiffness that a shorter-chains polymer imparts to the material, as no deentanglement of chains occurs during extension. When using the high molecular weight polymer, the work of fracture is higher and the composite can reach strains 10 times larger (around 100\%).

Upon fracture or damage, the "solid-liquid" polymer is released and flows to reform the graphene network and the composite heals to recover its mechanical and electrical properties (Figs. $1 \mathrm{~b}$ and 1c). Damage at different levels from surface to deep scratches or complete ruptures can be efficiently repaired. Cut samples can autonomously heal and fully recover their mechanical and electrical properties by simply placing their surfaces in contact without additional pressure (Fig. 3). In contrast to other materials [13], no external stimulus, i.e. temperature or pressure, is required for self-healing. Samples broken in tension recover autonomously above $80 \%$ of their strength by placing the fracture surfaces in contact at ambient conditions for 10 minutes (Fig. 3a). Complete recovery is achieved in only 1 day. Electrical conductivity is also recovered after cutting the sample in half and subsequently putting the parts back together without additional pressure. The composites recover above $90 \%$ of their original conductivity in only a few minutes (Fig. 3d).

The polymer wets rGO facilitating healing, and the graphene flakes exposed during fracture (Fig. 3c) re-connect and quickly restore the network's electrical conductivity. Once healing is achieved, the polymer is re-encapsulated in the graphene scaffold to form a robust, solid composite. Since healing is driven by polymer flow and by the dynamic dative bond driven interactions between polymer chains (Fig. 3b), the healing agent does not get exhausted. Self-repair 
can be repeated multiple times; we have performed 6 to 8 healing cycles in the experiments reported in this work with no loss of structural healing capabilities. Unlike many other materials structural healing is not reduced after exposing the fracture surfaces to the environment for long times. Surfaces left exposed for over 1 day could heal as much as freshly cut material.

The mechanical and electrical properties of the self-healing composites allow the preparation of bulk materials and films able to sense deformation and mechanical forces, i.e. tension, pressure and flexion (Fig. 4). Sensing is based on the measurable variation of voltage/resistance with strain. A tensile-electrical test has been carried out to analyze the strain at which the sample loses its conductivity. The rGO scaffold stretches with the polymer and manages to still be conductive at strains up to $30 \%$. Fracture of the composite coincides with the break of the conductive network (Fig. 4a) suggesting that the graphene scaffold in the composite can strain much more than before infiltration with PBS. It could be deduced that the polymer enables the rearrangement of the graphene flakes in the scaffold walls during tension without losing their connection.

Tactile pressure sensitivity can be monitored using a cyclic compression test while measuring the voltage variations in a 4-point probe configuration. Under a compression of $\sim 200 \mathrm{kPa}$, the measured voltage becomes more that two times higher. Buckling and deformation of the graphene network increase the length of the conductive path. Upon release the voltage gradually decreases in 10 to 20 seconds to the initial value. The small increase in voltage at zero pressure after the first cycles relates to the residual non-elastic deformation of the composites (Fig. 4b). However, after only a few cycles (2-3) the system becomes stable, voltage drops to the same baseline values and pressure measurements remain 
reproducible. This data suggests that deformation of the graphene network in the composite is highly reversible, as it has also been observed for the starting graphene scaffolds ${ }^{[14]}$.
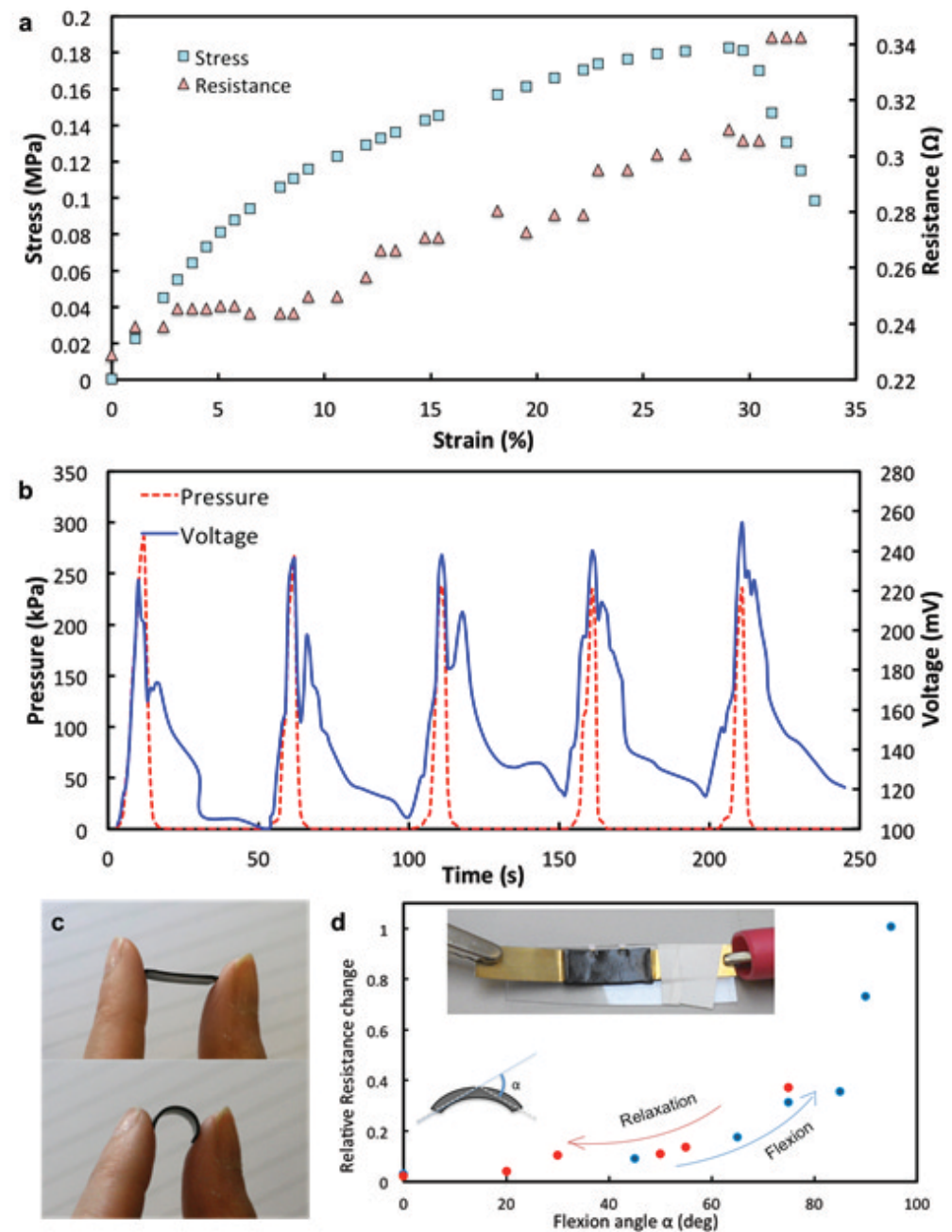

Figure 4: Self-healing composite, sensing properties. (a) Tensile-electric test recording the variation of resistance (in 2-point probe configuration) with strain. The sample is still conductive after strains of $30 \%$. Fracture and loss of electrical conductivity happen simultaneously. (b) Pressure sensitivity. The voltage variation (in 4-point probe configuration) is monitored under cyclic compression tests as a function of time. The composite has a graphene content of $0.5 \mathrm{wt} . \%$ and is filled with a LMw polymer. Electrode distance $1.5 \mathrm{~mm}$, sample length across $6.4 \mathrm{~mm}$ and sample cross-sectional area $56 \mathrm{~mm}^{2}$. (c) Flexible films formed by the slicing of bulk composites into tapes less than $500 \mu \mathrm{m}$ thick. The films are self-standing. (d) Variation of resistance in 2-point probe configuration of a $500 \mu$ m-thick film flexed above $90 \mathrm{deg}$. The graph shows the effect of flexion and relaxation and the results have been repeated for 3 cycles without noticeable variations. The film recovers its initial resistivity after relaxation. 
It is possible to use the material to prepare freestanding and flexible composite films with thicknesses below $500 \mu \mathrm{m}$ (Fig. 4c). These films can fully recover their shape from bending after the removal of external stress, and their electrical resistance depends on the degree of flexion, showing promise for flexion sensing applications. The resistance increases under flexion, however it goes back to lower values once the film is brought to the initial configuration. The cycle is fully repeatable (Fig. 4d).

For natural and synthetic materials alike, one of the key issues is the critical volume of the flaw above which repair is not possible. In this respect, our results illustrate the advantages of encapsulating a polymer able to flow at low stresses. However, to assure complete healing and large critical volumes we should maximize the healant content while retaining structural stability. The graphene network allows us to form a solid composite containing up to 99.8 wt $\%$ of a supramolecular polymer that behaves as a liquid at low strain rates. After damage the polymer is released and due to its "solid-liquid" behavior flows to heal deep scratches or re-join fracture surfaces and reform the conductive network at room temperature. Due to its dynamically-bonded nature the healing polymer is never exhausted and can act multiple times providing repeatable, autonomous recovery of mechanical and electrical properties. The mechanical properties of the material allow the fabrication of bulk parts and films whose electrical conductivities can reach values up to $90 \mathrm{~S} \mathrm{~m}^{-1}$ and that are sensitive to pressure and flexion. Being able to heal and sense external forces and deformation as a "synthetic skin", these materials could be of interest the biomedical, robotic and energy fields. 


\section{Methods}

Graphene oxide was prepared following the method described in [15] and its main properties are summarized in [16], Graphene oxide suspensions in water with concentrations ranging from 15 to $30 \mathrm{mg} \mathrm{cm}^{-3}$ were prepared using sucrose and polyvinyl alcohol (PVA) as additives (the GO:additive ratio was $1: 2$ in weight). The slurry was subsequently freeze-casted at a rate of $10 \mathrm{~K} \mathrm{~min}$ 1 and freeze dryed in a Labconco FreeZone $4.5 \mathrm{~L}$ console. The scaffolds obtained were reduced at $950^{\circ} \mathrm{C}$ for 20 minutes in $10 \% \mathrm{H} 2 / 90 \% \mathrm{Ar}$ atmosphere inside a tubular oven. Poly(dimethylsiloxane) (PDMS) (25cSt and 65cSt Aldrich () and Boron Oxide nanoparticles $\left(\mathrm{B}_{2} \mathrm{O}_{3}\right.$ SkySpring Nanomaterials, Inc, average size $80 \mathrm{~nm}$ ) were vigorously mixed together in concentrations varying from 1 to $5 w t \%$, depending on the molecular weight of the polymer used, and infiltrated via vacuum casting inside the porous rGO newtorks with cylindrical (Vol 3.14 $\left.\mathrm{cm}^{3}\right)$ or parallelepiped $\left(\right.$ Vol 16 $\left.\mathrm{cm}^{3}\right)$ shape. The crosslinking reaction was carried out in the oven at $200{ }^{\circ} \mathrm{C}$ for 6 hours. For rheological tests a DHR3 rheometer from TA Instruments was used in both frequency swipe mode and amplitude swipe mode. For the samples with dispersed graphene flakes, reduced (as for the scaffolds) graphene oxide powder (obtained as described previously) was dispersed in PDMS, with a concentrations up to $10 \mathrm{wt} \%$, via ultrasonication tip (UP200S SciMed, UK) for $1 \mathrm{~h}$ with set amplitude of $50 \%$ and cycle 0.5 . The solution was then cross-linked as described above.

The composite samples were cut into dog-bone shape, with internal crosssectional area of $\sim 0.12 \mathrm{~cm}^{2}$, for tensile testing and tested using Zwick Roell mechanical testing machines, used for compression testing as well and 
operated in constant displacement. The conductivity of the sample was monitored in 2 point via benchtop multimeter and in 4-point probe configuration by using a power supply unit (PSU) with constant input current of $0.08 \mathrm{~A}$ and a standard 4 channels bench multimeter to measure the voltage variation. For the electrical tests cylindrical samples were used as prepared and contacted to the circuit via copper electrodes. Films were cut out of parallelepiped (approximately $0.5 \mathrm{~mm} \times 30 \mathrm{~mm} \times 2 \mathrm{~mm}$ ) structures and used in flexion conductivity tests by connecting the two opposite ends to the multimeter through flexible copper foil cut to shape. The films were bent manually while constantly monitoring the conductivity change with bending degree through a 30 fps camera. Samples for imaging were prepared by coating the surface with a $5 \mathrm{~nm}$ chromium layer and characterized at $15 \mathrm{keV}$ in a Zeiss CrossBeam workstation Auriga SEM.

Due to the anisotropic structure of the starting graphene network there is some expected dependence of the mechanical and electrical performance on the testing direction. The strength and conductivity measurements were taken in the direction parallel to the channels in the graphene structure (the direction of ice growth during freeze casting). Although the measurement direction may affect some of the absolute values (see the supplementary information), the variation is relatively small and the observed trends and healing behavior remain the same.

\section{Acknowledgements}

The authors would like to acknowledge the EPSRC grant Graphene 3D Networks (EP/K01658X/1). S.B. and V.G.R. would like to acknowledge the European Commission (FP7 Marie Curie Intra-European Fellowships ACIN and GRAPES). 
ED would like to acknowledge EPSRC-DTA funding. N.N. would like to

acknowledge EPSRC grant Engineering with Graphene for Multifunctional

Coatings and Fiber-Composites EP/K016792/1.

\section{References}

[1] K. S. Toohey, N. R. Sottos, J. A. Lewis, J. S. Moore, S. R. White, Nat Mater 2007, 6, 581.

[2] B. C. K. Tee, C. Wang, R. Allen, Z. N. Bao, Nat Nanotechnol 2012, 7, 825; C. Y. Hou, T. Huang, H. Z. Wang, H. Yu, Q. H. Zhang, Y. G. Li, Sci Rep-Uk 2013, 3.

[3] N. Holten-Andersen, M. J. Harrington, H. Birkedal, B. P. Lee, P. B. Messersmith, K. Y. C. Lee, J. H. Waite, P Natl Acad Sci USA 2011, 108, 2651; P. Cordier, F.

Tournilhac, C. Soulie-Ziakovic, L. Leibler, Nature 2008, 451, 977; X. X. Chen, M. A. Dam, K. Ono, A. Mal, H. B. Shen, S. R. Nutt, K. Sheran, F. Wudl, Science 2002, 295, 1698; M. Burnworth, L. M. Tang, J. R. Kumpfer, A. J. Duncan, F. L. Beyer, G. L. Fiore, S. J. Rowan, C. Weder, Nature 2011, 472, 334.

[4] M. W. Keller, S. R. White, N. R. Sottos, Adv Funct Mater 2007, 17, 2399.

[5] M. M. Caruso, B. J. Blaiszik, S. R. White, N. R. Sottos, J. S. Moore, Adv Funct Mater 2008, 18, 1898.

[6] G. M. L. van Gemert, J. W. Peeters, S. H. M. Sontjens, H. M. Janssen, A. W. Bosman, Macromol Chem Phys 2012, 213, 234; R. Martin, A. Rekondo, A. R. de Luzuriaga, G. Cabanero, H. J. Grande, I. Odriozola, J Mater Chem A 2014, 2, 5710; A. L. Litvin, V. N. Bliznyuk, V. V. Tsukruk, S. Valiyaveettil, D. L. Kaplan, Abstr Pap Am Chem S 1996, 212, 273.

[7] M. P. Goertz, X. Y. Zhu, J. E. Houston, J Polym Sci Pol Phys 2009, 47, 1285.

[8] J. Li, J. K. Kim, Compos Sci Technol 2007, 67, 2114.

[9] S. Deville, E. Saiz, R. K. Nalla, A. P. Tomsia, Science 2006, 311, 515.

[10] X. F. Li, D. A. Zhang, K. W. Xiang, G. S. Huang, Rsc Adv 2014, 4, 32894.

[11] A. Juhász, P. Tasnádi, L. Fábry, Physics Education 1984, 19, 302.

[12] Z. Liu, S. J. Picken, N. A. M. Besseling, Macromolecules 2014, 47, 4531.

[13] D. Y. Wu, S. Meure, D. Solomon, Prog Polym Sci 2008, 33, 479.

[14] S. Barg, F. M. Perez, N. Ni, P. D. V. Pereira, R. C. Maher, E. Garcia-Tunn, S. Eslava, S. Agnoli, C. Mattevi, E. Saiz, Nat Commun 2014, 5.

[15] D. C. Marcano, D. V. Kosynkin, J. M. Berlin, A. Sinitskii, Z. Z. Sun, A. Slesarev, L. B. Alemany, W. Lu, J. M. Tour, Acs Nano 2010, 4, 4806.

[16] E. Garcia-Tuñon, S. Barg, J. Franco, R. Bell, S. Eslava, E. D'Elia, R. C. Maher, F. Guitian, E. Saiz, Adv Mater 2015, 27, 1688. 